\title{
The Fox property for codimension one embeddings of products of three spheres into spheres
}

\author{
LAÉRCIO APARECIDO LUCAS \\ OSAMU SAEKI
}

\begin{abstract}
Fox has shown that for every closed connected surface smoothly embedded in $S^{3}$, the closure of each component of its complement is diffeomorphic to the closure of the complement of a handlebody embedded in $S^{3}$. In this paper, we study a similar "Fox property" for smooth embeddings of $S^{p} \times S^{q} \times S^{r}$ in $S^{p+q+r+1}$.
\end{abstract}

57R40; 57Q45

\section{Introduction}

For positive integers $p, q$ and $r$, let $\Sigma^{p, q} \subset S^{p+q+1}$ be the boundary of a tubular neighborhood of $S^{p}$ standardly embedded in $S^{p+q+1}$. Note that $\Sigma^{p, q} \cong S^{p} \times S^{q}$. Consider $S^{p+q+1}$ to be standardly embedded in $S^{p+q+r+1}$. Let $N_{+}^{p, q, r}\left(\cong S^{p} \times\right.$ $S^{q} \times D^{r+1}$ ) be a tubular neighborhood of $\Sigma^{p, q}$ in $S^{p+q+r+1}$, and $N^{p, q, r}$ be the closure of $S^{p+q+r+1} \backslash N_{+}^{p, q, r}$. The main result of this paper is the following.

Theorem 1.1 If $1 \leq q \leq r, 3 \leq q+r$, and either $r \neq q+1$ or $r=q+1$ is even, then for each smooth embedding $f: S^{1} \times S^{q} \times S^{r} \rightarrow S^{q+r+2}$, there exist a pair of smooth embeddings $f_{+}: N_{+}^{p^{\prime}, q^{\prime}, r^{\prime}} \rightarrow S^{q+r+2}$ and $f_{-}: N^{p^{\prime}}, q^{\prime}, r^{\prime} \rightarrow S^{q+r+2}$ for some permutation $\left(p^{\prime}, q^{\prime}, r^{\prime}\right)$ of $(1, q, r)$ such that the closures of the connected components of $S^{q+r+2} \backslash \operatorname{Im} f$ are diffeomorphic to the closures of $S^{q+r+2} \backslash \operatorname{Im} f_{+}$ and $S^{q+r+2} \backslash \operatorname{Im} f_{-}$.

Theorem 1.1 can be considered as an analogue of the following classical result of Fox. Let $F$ be a closed connected orientable surface. The image of a standard embedding $F \rightarrow S^{3}$ splits $S^{3}$ into two handlebodies. Fox [3] has shown that for every smooth embedding $f: F \rightarrow S^{3}$, the closure of each of the two components of $S^{3}-f(F)$ is diffeomorphic to the closure of the complement of a handlebody embedded in $S^{3}$.

Katanaga and the second author [5] have formulated a "Fox property" for dimension four and we now extend this to higher dimensions. Using the notation that we shall use in 
Section 1, let $M$ be a smooth closed connected (n-1)-dimensional manifold for which a set of "standard embeddings" $f_{\sigma}: M \rightarrow S^{n}, \sigma \in \mathcal{S}$, is given. Furthermore, denote by $N_{+}^{\sigma}$ and $N_{-}^{\sigma}$ the closures of the two components of $S^{n} \backslash f_{\sigma}(M)$. (It is often the case that $N_{ \pm}^{\sigma}$ are regular neighborhoods of some lower-dimensional polyhedra embedded in $S^{n}$ with their boundaries smoothed.) Then a smooth embedding $f: M \rightarrow S^{n}$ is said to have the Fox property if, for some $\sigma \in \mathcal{S}$, there exist a pair of smooth embeddings $f_{+}: N_{+}^{\sigma} \rightarrow S^{n}$ and $f_{-}: N_{-}^{\sigma} \rightarrow S^{n}$ such that the closures of the two components of $S^{n} \backslash f(M)$ are diffeomorphic to the closures of $S^{n} \backslash \operatorname{Im} f_{+}$and $S^{n} \backslash \operatorname{Im} f_{-}$. We can define the same concept in the TOP and the PL categories as well, where we always impose the condition that the embeddings should be locally flat. Note that in the literature, some examples of manifolds whose codimension one embeddings always have the Fox property are known. Embeddings of spheres $S^{n-1}$ in $S^{n}$ have the Fox property according to the generalized Schönflies theorem (Alexander [1], Brown [2] and Smale [14]), where in the case $n=4$ we work in the TOP category because of the still-unsolved smooth and PL Schönflies problems. Embeddings of products of two spheres $S^{p} \times S^{q}$ in $S^{p+q+1}$ (Alexander [1], Goldstein [4], Kosiński [8], Neto and the authors [9], Rubinstein [13] and Wall [17]), embeddings of the quaternion space in $S^{4}$ (Katanaga and the second author [5]) and also of the 3-dimensional torus $S^{1} \times S^{1} \times S^{1}$ in $S^{4}$ (the authors [12]) all have the Fox property, where again we need to work in the TOP category for $n=4$ because of the unsolved Schönflies problems.

In view of Theorem 1.1, we can say that every smooth embedding of $S^{1} \times S^{q} \times S^{r}$ into $S^{q+r+2}, 1 \leq q \leq r, 3 \leq q+r$, has the Fox property, provided $r \neq q+1$ or $r=q+1$ is even.

We can also define a stronger property as follows. The Alexander torus theorem [1] states that every smoothly embedded torus in $S^{3}$ bounds a solid torus. Generalizing this result, we say that a smooth embedding $f: M \rightarrow S^{n}$ has the Alexander property if the closure of one of the two components of $S^{n} \backslash f(M)$ is diffeomorphic to $N_{+}^{\sigma}$ or $N_{-}^{\sigma}$ for some $\sigma \in \mathcal{S}$. Embeddings of spheres and products of two spheres all in fact have the Alexander property (with the usual proviso about dimension 4).

Note that the Alexander property implies the Fox property. This can be seen as follows. Suppose that the closure $C$ of a component of $S^{n} \backslash f(M)$ is diffeomorphic to $N_{+}^{\sigma}$. Then, let $f_{-}: N_{-}^{\sigma} \rightarrow S^{n}$ be the standard embedding, while we set $f_{+}: N_{+}^{\sigma} \rightarrow S^{n}$ to be the composition of a diffeomorphism $N_{+}^{\sigma} \cong C$ and the inclusion $C \hookrightarrow S^{n}$. Then, we see that the closures of the components of $S^{n} \backslash f(M)$ are diffeomorphic to the closures of $S^{n} \backslash \operatorname{Im} f_{+}$and $S^{n} \backslash \operatorname{Im} f_{-}$. 
In the case of $S^{p} \times S^{q} \times S^{r}$, an embedding $S^{p} \times S^{q} \times S^{r} \rightarrow S^{p+q+r+1}$ is standard $^{1}$ if its image coincides with the boundary of a tubular neighborhood of a product of two spheres standardly embedded in $S^{p+q+r+1}$, or more precisely, if its image is isotopic to $\partial N_{+}^{p^{\prime}, q^{\prime}, r^{\prime}}=\partial N^{p^{\prime}}, q^{\prime}, r^{\prime}\left(\cong S^{p^{\prime}} \times S^{q^{\prime}} \times S^{r^{\prime}}\right)$ for some permutation $\left(p^{\prime}, q^{\prime}, r^{\prime}\right)$ of $(p, q, r)$. In [11], the authors have shown that if $2 \leq p \leq q \leq r$ and either $p+q \neq r$ or $p+q=r$ is even, then every smooth embedding $f: S^{p} \times S^{q} \times S^{r} \rightarrow S^{p+q+r+1}$ has the Alexander property. Furthermore, for $1 \leq p \leq q \leq r$ with $r=p+q$ odd, we have constructed infinitely many smooth embeddings $f_{n}: S^{p} \times S^{q} \times S^{r} \rightarrow S^{p+q+r+1}$, $n \in \mathbb{Z} \backslash\{0\}$, which do not have the Alexander property. In this paper we will show that in fact, they do not have the Fox property, provided $n \neq 0,-1$ (see Theorem 3.2 in Section 3). This means that the embeddings $f_{n}, n \neq 0,-1$, constructed in [11] are highly knotted. This answers the question posed at the end of [12]. This also shows that Theorem 1.1 does not hold if $r=q+1$ is odd.

We warn the reader that in the literature, the terminology "Fox's property" has been used in different contexts (for example Kinoshita [7] and Suzuki [15]). In this paper, we always use the terminology "Fox property" in the sense defined above.

The paper is organized as follows. In Section 2, we prove Theorem 1.1. In Section 3, we recall the construction of the embeddings $f_{n}$ mentioned above which do not have the Alexander property, and state Theorem 3.2. In Section 4, we prepare a lemma concerning diffeomorphisms of $S^{p} \times S^{q} \times S^{p+q}$, which we will use in Section 5 to show that in fact the embeddings $f_{n}$ do not have the Fox property. In Section 6 , we summarize the results obtained so far and pose some questions. Note that Theorems 1.1 and 3.2 are almost independent of each other, so that if the reader is interested only in Theorem 3.2, Section 2 may safely be skipped.

Throughout the paper all homology and cohomology groups are with coefficients in $\mathbb{Z}$. The symbol " $[*]$ " denotes the homology class represented by $*$. For a space $X$, "id $X$ " denotes the identity map of $X$. The exterior of an embedding (or of its image) is the closure of the complement to the regular (or tubular) neighborhood of the image of the embedding.

\section{Proof of Theorem 1.1}

Let $f: S^{1} \times S^{q} \times S^{r} \rightarrow S^{q+r+2}$ be an embedding. Let $C_{1}$ and $C_{2}$ be the closures of the two components of $S^{q+r+2} \backslash \operatorname{Im} f$. Our strategy for the proof of Theorem 1.1 is to show that $f$ has the Alexander property, or to proceed as follows.

\footnotetext{
${ }^{1}$ Unfortunately, the explanation given by the authors in [11, Definition 8.1] needs to be corrected.
} 
(1) Construct closed manifolds $X_{j}=C_{j} \cup N_{ \pm}^{p^{\prime}, q^{\prime}, r^{\prime}}, j=1$, 2, for some permutation $\left(p^{\prime}, q^{\prime}, r^{\prime}\right)$ of $(1, q, r)$.

(2) Show that $X_{j}$ are homotopy spheres.

(3) Then, show that $X_{j}$ may be assumed to be diffeomorphic to the standard sphere $S^{q+r+2}$.

We can then conclude that $C_{j}$ are as required. Note that the above Step (2) is nontrivial, although the proof only uses standard homology calculations and the van Kampen theorem.

Proof of Theorem 1.1 In the following, $f: S^{1} \times S^{q} \times S^{r} \rightarrow S^{q+r+2}$ is an arbitrary smooth embedding with $1 \leq q \leq r,(q, r) \neq(1,1)$, and either $r \neq q+1$ or $r=q+1$ is even. We denote by $C_{1}$ and $C_{2}$ the closures of the two components of $S^{q+r+2} \backslash \operatorname{Im} f$. We identify $\partial C_{1}=\partial C_{2}$ with $S^{1} \times S^{q} \times S^{r}$ by $f$, and $i_{j}: S^{1} \times S^{q} \times S^{r} \rightarrow C_{j}$, $j=1,2$, denote the inclusion maps.

We divide the proof into 5 cases according to the values of $q$ and $r$, and we subdivide these into a total of 10 subcases according to the homologies of $C_{1}$ and $C_{2}$. In Case (1-1), $f$ has the Alexander property. In Cases (1-2), (2-1), (4-1), (5-1) and (5-2), we will use standard diffeomorphisms for attaching $C_{i}$ and $N_{ \pm}$. In Cases (2-2), (3-1), (3-2) and (4-2), we need to choose particular diffeomorphisms for the attachment. The key cases are, therefore, (1-1), (1-2) and (2-2), in which the reader can find the main ideas of proof.

Case $11<q<r$ and $r \neq q+1$

By Alexander duality, we can show that either $C_{1}$ or $C_{2}$ has the same homology as $S^{1} \times S^{q}, S^{1} \times S^{r}$ or $S^{q} \times S^{r}$ (for details, see the argument in [11, Section 2]). In the following, we assume that $C_{1}$ has such a homology.

(1-1) If $H_{*}\left(C_{1}\right) \cong H_{*}\left(S^{q} \times S^{r}\right)$, then by [12, Proposition 1.2 (i)], $C_{1}$ is diffeomorphic to $D^{2} \times S^{q} \times S^{r}$. Consequently $f$ has the Alexander property and hence the Fox property.

(1-2) Suppose $H_{*}\left(C_{1}\right) \cong H_{*}\left(S^{1} \times S^{q}\right)$ or $H_{*}\left(S^{1} \times S^{r}\right)$. In the following, we assume $H_{*}\left(C_{1}\right) \cong H_{*}\left(S^{1} \times S^{q}\right)$ (the other case can be treated similarly). By the same argument as in [11, Section 2], we see that the natural inclusion

$$
i_{1}^{\prime}: S^{1} \times S^{q} \times\{*\} \rightarrow S^{1} \times S^{q} \times S^{r}=\partial C_{1} \stackrel{i_{1}}{\longrightarrow} C_{1}
$$


induces a homology equivalence.

Let $\theta_{1}: \partial C_{2}=S^{1} \times S^{q} \times S^{r} \rightarrow \partial\left(S^{1} \times S^{q} \times D^{r+1}\right)$ be the diffeomorphism obtained by the natural identification. Let us first show that $X_{1}=\left(S^{1} \times S^{q} \times D^{r+1}\right) \cup_{\theta_{1}} C_{2}$, obtained by gluing $S^{1} \times S^{q} \times D^{r+1}$ and $C_{2}$ by $\theta_{1}$ along the boundaries, is a homotopy $(q+r+2)$-sphere.

By an argument using the van Kampen theorem similar to that in the proof of [12, Theorem 1.3], we can show that $X_{1}$ is simply connected. (The Hurewicz homomorphism $\pi_{1}\left(C_{1}\right) \rightarrow H_{1}\left(C_{1}\right) \cong \pi_{1}\left(S^{1} \times S^{q} \times D^{r+1}\right)$ and the identity $\pi_{1}\left(C_{2}\right) \rightarrow \pi_{1}\left(C_{2}\right)$ induce an epimorphism $\pi_{1}\left(S^{q+r+2}\right) \rightarrow \pi_{1}\left(X_{1}\right)$.)

Let us consider the following commutative diagram

$$
\begin{array}{cc}
H_{*}\left(S^{1} \times S^{q} \times S^{r}\right) \stackrel{\left(i_{1 *},-i_{2 *}\right)}{\longrightarrow} & H_{*}\left(C_{1}\right) \oplus H_{*}\left(C_{2}\right) \\
\text { id } \downarrow & \downarrow \rho \oplus \text { id }
\end{array}
$$

where $j_{1}: S^{1} \times S^{q} \times S^{r} \rightarrow S^{1} \times S^{q} \times D^{r+1}$ is the inclusion map, $\rho: H_{*}\left(C_{1}\right) \rightarrow$ $H_{*}\left(S^{1} \times S^{q} \times D^{r+1}\right)$ is the isomorphism obtained by the composition

$$
\begin{aligned}
& H_{*}\left(C_{1}\right) \stackrel{\left(i_{1 *}^{\prime}\right)^{-1}}{\longrightarrow} H_{*}\left(S^{1} \times S^{q} \times\{*\}\right) \stackrel{\left(\left.\theta_{1}\right|_{S^{1} \times S^{q_{\times\{*}}}\right)_{*}^{-1}}{\longrightarrow} H_{*}\left(S^{1} \times S^{q} \times\{*\}\right) \\
& \stackrel{j_{1 *}^{\prime}}{\longrightarrow} H_{*}\left(S^{1} \times S^{q} \times D^{r+1}\right),
\end{aligned}
$$

and $j_{1}^{\prime}: S^{1} \times S^{q} \times\{*\} \rightarrow S^{1} \times S^{q} \times D^{r+1}$ is the inclusion map. Then, by comparing the Mayer-Vietoris exact sequences for $\left(C_{1}, C_{2}\right)$ and $\left(S^{1} \times S^{q} \times D^{r+1}, C_{2}\right)$, we see that $X_{1}$ has the same homology as $S^{q+r+2}$. Therefore, $X_{1}$ is a homotopy $(q+r+2)-$ sphere.

By taking connected sum with the inverse of $X_{1}$ (in the group of homotopy spheres) if necessary, we can arrange that $X_{1}$ is diffeomorphic to the standard $(q+r+2)-$ sphere, since $q+r+2 \geq 5$ (for details, see Kervaire and Milnor [6]). Therefore, $C_{2}$ is diffeomorphic to the exterior of a smooth embedding of $S^{1} \times S^{q} \times D^{r+1}$ into $S^{q+r+2}$.

Now, let $N_{-}$be the exterior of the standard embedding of $S^{1} \times S^{q} \times D^{r+1}$ into $S^{q+r+2}$ (see also Section 3). For the natural identification map $\theta_{2}: \partial N_{-}=S^{1} \times S^{q} \times S^{r} \rightarrow \partial C_{1}$, let us show that $X_{2}=C_{1} \cup_{\theta_{2}} N_{-}$is a homotopy $(q+r+2)$-sphere.

First, note that by Alexander duality we have

$$
H_{*}\left(C_{2}\right) \cong H_{*}\left(N_{-}\right) \cong H_{*}\left(S^{r+1} \vee S^{q+r} \vee S^{r}\right) \text {. }
$$


Let $j_{2}: S^{1} \times S^{q} \times S^{r} \rightarrow N_{-}$be the inclusion map. Note that $1, q, q+1, r, r+1, q+r$ are all distinct.

Let us consider the Mayer-Vietoris exact sequences for the pairs $\left(C_{1}, C_{2}\right)$ and $\left(S^{1} \times\right.$ $\left.S^{q} \times D^{r+1}, N_{-}\right)$, respectively

$$
\begin{aligned}
H_{k+1}\left(S^{q+r+2}\right) & \rightarrow H_{k}\left(S^{1} \times S^{q} \times S^{r}\right) \rightarrow H_{k}\left(C_{1}\right) \oplus H_{k}\left(C_{2}\right) \rightarrow H_{k}\left(S^{q+r+2}\right), \\
H_{k+1}\left(S^{q+r+2}\right) \rightarrow H_{k}\left(S^{1} \times\right. & \left.S^{q} \times S^{r}\right) \\
& \rightarrow H_{k}\left(S^{1} \times S^{q} \times D^{r+1}\right) \oplus H_{k}\left(N_{-}\right) \rightarrow H_{k}\left(S^{q+r+2}\right) .
\end{aligned}
$$

We see that $i_{2 *}: H_{k}\left(S^{1} \times S^{q} \times S^{r}\right) \rightarrow H_{k}\left(C_{2}\right)$ and $j_{2 *}: H_{k}\left(S^{1} \times S^{q} \times S^{r}\right) \rightarrow H_{k}\left(N_{-}\right)$ are isomorphisms for $k=r, r+1, q+r$. Therefore, we can construct an isomorphism $\tau: H_{*}\left(C_{2}\right) \rightarrow H_{*}\left(N_{-}\right)$such that the diagram

$$
\begin{array}{cc}
H_{*}\left(S^{1} \times S^{q} \times S^{r}\right) \stackrel{\left(i_{1 *},-i_{2 *}\right)}{\longrightarrow} & H_{*}\left(C_{1}\right) \oplus H_{*}\left(C_{2}\right) \\
\text { id } \downarrow & \downarrow \text { id } \oplus \tau \\
H_{*}\left(S^{1} \times S^{q} \times S^{r}\right) \stackrel{\left(i_{1 *},-j_{2 *}\right)}{\longrightarrow} H_{*}\left(C_{1}\right) \oplus H_{*}\left(N_{-}\right)
\end{array}
$$

is commutative. Hence, $X_{2}$ has the same homology as the $(q+r+2)$-sphere. Furthermore, we can show that $X_{2}$ is simply connected as before. Hence, $X_{2}$ is a homotopy $(q+r+2)$-sphere. Then, by modifying $\theta_{2}$ appropriately, we can arrange so that $X_{2}$ is diffeomorphic to the standard $(q+r+2)$-sphere. Therefore, $C_{1}$ is diffeomorphic to the exterior of a smooth embedding of $N_{-}$into $S^{q+r+2}$.

Consequently, $f$ has the Fox property when $1<q<r$ and $r \neq q+1$.

Case $21=q<r \neq 2$

By the same argument as in [11, Section 4], we have only to consider the two cases

$$
\begin{aligned}
& H_{*}\left(C_{1}\right) \cong H_{*}\left(S^{1} \times S^{1}\right), \\
& H_{*}\left(C_{1}\right) \cong H_{*}\left(S^{1} \times S^{r}\right) .
\end{aligned}
$$

(2-1) Suppose $H_{*}\left(C_{1}\right) \cong H_{*}\left(S^{1} \times S^{1}\right)$. By the same argument as in [11, Section 4], we see that the natural inclusion $S^{1} \times S^{1} \times\{*\} \rightarrow C_{1}$ induces a homology equivalence. Then, by the same argument as in Case 1 , we can show that $X_{1}=\left(S^{1} \times S^{1} \times D^{r+1}\right) \cup_{\theta_{1}}$ $C_{2}$ is diffeomorphic to the standard $(r+3)$-sphere for some attaching diffeomorphism $\theta_{1}$.

Let us consider $X_{2}=C_{1} \cup_{\theta_{2}} N_{-}$, where $\theta_{2}: \partial N_{-}=S^{1} \times S^{1} \times S^{r} \rightarrow \partial C_{1}$ is the natural identification map, and $N_{-}\left(\simeq S^{r+1} \vee S^{r+1} \vee S^{r}\right.$, see Section 3$)$ is the exterior of the 
standard embedding of $S^{1} \times S^{1} \times D^{r+1}$ into $S^{r+3}$. Let us consider the Mayer-Vietoris exact sequence for the pair $\left(C_{1}, C_{2}\right)$

$$
H_{k+1}\left(S^{r+3}\right) \rightarrow H_{k}\left(S^{1} \times S^{1} \times S^{r}\right) \rightarrow H_{k}\left(C_{1}\right) \oplus H_{k}\left(C_{2}\right) \rightarrow H_{k}\left(S^{r+3}\right) .
$$

Since the inclusion $S^{1} \times S^{1} \times\{*\} \rightarrow C_{1}$ induces a homology equivalence, we see that $i_{2 *}: H_{k}\left(S^{1} \times S^{1} \times S^{r}\right) \rightarrow H_{k}\left(C_{2}\right)$ is an isomorphism for $k=r, r+1$. Therefore, by the same argument as in Case (1-2), we can show that $X_{2}$ is a homotopy $(r+3)$-sphere.

Hence $f$ has the Fox property.

(2-2) Suppose $H_{*}\left(C_{1}\right) \cong H_{*}\left(S^{1} \times S^{r}\right)$. Let us consider the exact sequence

$$
0 \rightarrow \operatorname{Ker} i_{1 *} \rightarrow H_{1}\left(\partial C_{1}\right) \stackrel{i_{1 *}}{\longrightarrow} H_{1}\left(C_{1}\right) \rightarrow H_{1}\left(C_{1}, \partial C_{1}\right) .
$$

Since $H_{1}\left(\partial C_{1}\right) \cong \mathbb{Z} \oplus \mathbb{Z}, H_{1}\left(C_{1}\right) \cong \mathbb{Z}$ and $H_{1}\left(C_{1}, \partial C_{1}\right) \cong H^{r+2}\left(C_{1}\right)=0$, there exists a base $\left\{\gamma_{1}, \gamma_{2}\right\}$ of $H_{1}\left(\partial C_{1}\right) \cong \mathbb{Z} \oplus \mathbb{Z}$ such that $i_{1 *} \gamma_{2}=0$ and $i_{1 *} \gamma_{1}$ is a generator of $H_{1}\left(C_{1}\right) \cong \mathbb{Z}$.

Let

$$
\iota: S^{1} \times S^{1}=S^{1} \times S^{1} \times\{*\} \rightarrow S^{1} \times S^{1} \times S^{r}=\partial C_{1}
$$

be the natural inclusion. There exist $\gamma_{1}^{\prime}, \gamma_{2}^{\prime} \in H_{1}\left(S^{1} \times S^{1}\right)$ such that $\iota_{*} \gamma_{i}^{\prime}=\gamma_{i}, i=1,2$. Then there exists a diffeomorphism $\widehat{\theta}: S^{1} \times S^{1} \rightarrow S^{1} \times S^{1}$ such that $\widehat{\theta}_{*}\left[S^{1} \times\{*\}\right]=\gamma_{1}^{\prime}$ and $\widehat{\theta}_{*}\left[\{*\} \times S^{1}\right]=\gamma_{2}^{\prime}$. Set $\theta=\widehat{\theta} \times \operatorname{id}_{S^{r}}:\left(S^{1} \times S^{1}\right) \times S^{r} \rightarrow\left(S^{1} \times S^{1}\right) \times S^{r}$. Then by an argument similar to that in [11], we see that

$$
\left.i_{1} \circ \theta\right|_{S^{1} \times\{*\} \times S^{r}}: S^{1} \times\{*\} \times S^{r} \rightarrow \partial C_{1} \rightarrow C_{1}
$$

induces a homology equivalence.

By the universal coefficient theorem, we can show that it also induces an isomorphism of cohomology rings as well. Let $\eta_{1} \in H^{1}\left(C_{1}\right) \cong \mathbb{Z}$ and $\eta_{r} \in H^{r}\left(C_{1}\right) \cong \mathbb{Z}$ be generators. Then $\eta_{1} \smile \eta_{r}$ is a generator of $H^{r+1}\left(C_{1}\right)$ and we have

$$
\left\langle i_{1}^{*} \eta_{1}, \theta_{*}\left[S^{1} \times\{*\} \times\{*\}\right]\right\rangle= \pm 1,\left\langle i_{1}^{*} \eta_{1}, \theta_{*}\left[\{*\} \times S^{1} \times\{*\}\right]\right\rangle=0 .
$$

Hence $i_{1}^{*} \eta_{1}$ coincides with the Poincaré dual of $\theta_{*}\left[\{*\} \times S^{1} \times S^{r}\right] \in H_{r+1}\left(\partial C_{1}\right)$ up to sign. Furthermore, we see that $i_{1}^{*} \eta_{r}$ coincides with the Poincaré dual of $\theta_{*}\left[S^{1} \times\right.$ $\left.S^{1} \times\{*\}\right] \in H_{2}\left(\partial C_{1}\right)$. Therefore, the Poincaré dual of $i_{1}^{*}\left(\eta_{1} \smile \eta_{r}\right)$ coincides with $\theta_{*}\left[\{*\} \times S^{1} \times\{*\}\right] \in H_{1}\left(\partial C_{1}\right)$. This implies that

$$
\left\langle\eta_{1} \smile \eta_{r}, i_{1 *} \theta_{*}\left[\{*\} \times S^{1} \times S^{r}\right]\right\rangle=0,
$$

and hence $i_{1 *} \theta_{*}\left[\{*\} \times S^{1} \times S^{r}\right]=0$ in $H_{r+1}\left(C_{1}\right)$. 
Let us consider the Mayer-Vietoris exact sequence for the pair $\left(C_{1}, C_{2}\right)$

$$
H_{k+1}\left(S^{r+3}\right) \rightarrow H_{k}\left(S^{1} \times S^{1} \times S^{r}\right) \rightarrow H_{k}\left(C_{1}\right) \oplus H_{k}\left(C_{2}\right) \rightarrow H_{k}\left(S^{r+3}\right) .
$$

Since $i_{1 *} \gamma_{2}=0$ and $i_{1 *} \gamma_{1}$ is a generator of $H_{1}\left(C_{1}\right) \cong \mathbb{Z}, i_{2 *} \gamma_{2}$ must be a generator of $H_{1}\left(C_{2}\right) \cong \mathbb{Z}$. We also see that $i_{2 *} \theta_{*}\left[\{*\} \times S^{1} \times S^{r}\right]$ must be a generator of $H_{r+1}\left(C_{2}\right) \cong \mathbb{Z}$. Furthermore, $i_{2 *}: H_{2}\left(S^{1} \times S^{1} \times S^{r}\right) \rightarrow H_{2}\left(C_{2}\right)$ is an isomorphism.

Let $\theta_{1}: \partial C_{2}=S^{1} \times S^{1} \times S^{r} \rightarrow S^{1} \times S^{1} \times S^{r}$ be the diffeomorphism defined by $\theta_{1}=\theta^{-1}$ and set $X_{1}=\left(S^{1} \times D^{2} \times S^{r}\right) \cup_{\theta_{1}} C_{2}$. Then, by using the Mayer-Vietoris exact sequence

$$
H_{k+1}\left(X_{1}\right) \rightarrow H_{k}\left(S^{1} \times S^{1} \times S^{r}\right) \rightarrow H_{k}\left(S^{1} \times D^{2} \times S^{r}\right) \oplus H_{k}\left(C_{2}\right) \rightarrow H_{k}\left(X_{1}\right),
$$

we can show that $X_{1}$ is a homotopy $(r+3)$-sphere.

Let us consider $X_{2}=C_{1} \cup_{\theta_{2}} N_{-}$, where $N_{-}\left(\simeq S^{1} \vee S^{2} \vee S^{r+1}\right)$ is the exterior of the standard embedding of $S^{1} \times D^{2} \times S^{r}$ into $S^{r+3}$, and $\theta_{2}: \partial N_{-} \rightarrow \partial C_{1}$ is the diffeomorphism defined by $\theta_{2}=\theta$. Then, by the Mayer-Vietoris exact sequence

$$
H_{k+1}\left(X_{2}\right) \rightarrow H_{k}\left(S^{1} \times S^{1} \times S^{r}\right) \rightarrow H_{k}\left(C_{1}\right) \oplus H_{k}\left(N_{-}\right) \rightarrow H_{k}\left(X_{2}\right),
$$

we see that $X_{2}$ is also a homotopy $(r+3)$-sphere.

Therefore, $f$ has the Fox property.

Case $31<q=r$

By the same argument as in [11, Section 4], we have only to consider the two cases: $H_{*}\left(C_{1}\right) \cong H_{*}\left(S^{q} \times S^{q}\right)$ or $H_{*}\left(C_{1}\right) \cong H_{*}\left(S^{1} \times S^{q}\right)$. In the former case, the same argument as in (1-1) works. In the following, we suppose $H_{*}\left(C_{1}\right) \cong H_{*}\left(S^{1} \times S^{q}\right)$.

By the Mayer-Vietoris exact sequence

$$
H_{q+1}\left(S^{2 q+2}\right) \rightarrow H_{q}\left(S^{1} \times S^{q} \times S^{q}\right) \rightarrow H_{q}\left(C_{1}\right) \oplus H_{q}\left(C_{2}\right) \rightarrow H_{q}\left(S^{2 q+2}\right),
$$

we see that there exists a base $\left\{\gamma_{1}, \gamma_{2}\right\}$ of $H_{q}\left(S^{1} \times S^{q} \times S^{q}\right) \cong \mathbb{Z} \oplus \mathbb{Z}$ such that

$$
\begin{aligned}
& i_{1 * \gamma_{2}}=0, i_{1 * \gamma_{1}} \text { is a generator of } H_{q}\left(C_{1}\right) \cong \mathbb{Z}, \\
& i_{2 *} \gamma_{1}=0, i_{2 *} \gamma_{2} \text { is a generator of } H_{q}\left(C_{2}\right) \cong \mathbb{Z} .
\end{aligned}
$$

Let $\iota: S^{q} \times S^{q}=\{*\} \times S^{q} \times S^{q} \rightarrow S^{1} \times S^{q} \times S^{q}$ be the natural inclusion. Since $\iota_{*}: H_{q}\left(S^{q} \times S^{q}\right) \rightarrow H_{q}\left(S^{1} \times S^{q} \times S^{q}\right)$ is an isomorphism, we have $\gamma_{1}^{\prime}, \gamma_{2}^{\prime} \in H_{q}\left(S^{q} \times\right.$ $\left.S^{q}\right)$ such that $\iota_{*} \gamma_{i}^{\prime}=\gamma_{i}, i=1,2$. 
There exists a matrix

$$
\left(\begin{array}{ll}
a & c \\
b & d
\end{array}\right) \in \mathrm{GL}(2, \mathbb{Z})
$$

such that

$$
\begin{aligned}
& \gamma_{1}^{\prime}=a\left[S^{q} \times\{*\}\right]+b\left[\{*\} \times S^{q}\right], \\
& \gamma_{2}^{\prime}=c\left[S^{q} \times\{*\}\right]+d\left[\{*\} \times S^{q}\right] .
\end{aligned}
$$

(3-1) Let us first consider the case where $q \geq 3$ is odd. Set

$$
G_{1}=\left\{\left(\begin{array}{ll}
a_{11} & a_{12} \\
a_{21} & a_{22}
\end{array}\right) \in \operatorname{GL}(2, \mathbb{Z}) \mid a_{11} \equiv a_{22} \not \equiv a_{12} \equiv a_{21}(\bmod 2)\right\} .
$$

Then, we see that at least one of the following matrices lies in $G_{1}$ :

$$
\left(\begin{array}{ll}
a & c \\
b & d
\end{array}\right),\left(\begin{array}{ll}
a+c & c \\
b+d & d
\end{array}\right),\left(\begin{array}{ll}
a & a+c \\
b & b+d
\end{array}\right) .
$$

Then by Wall [16] (see also Golstein [4] and the authors [10]), there exists a diffeomorphism $\widehat{\theta}: S^{q} \times S^{q} \rightarrow S^{q} \times S^{q}$ which realizes one of the above matrices as the induced automorphism of $H_{q}\left(S^{q} \times S^{q}\right) \cong \mathbb{Z} \oplus \mathbb{Z}$ with respect to the base $\left\{\left[S^{q} \times\{*\}\right],\left[\{*\} \times S^{q}\right]\right\}$. For the diffeomorphism $\theta=\operatorname{id}_{S^{1}} \times \widehat{\theta}: S^{1} \times\left(S^{q} \times S^{q}\right) \rightarrow S^{1} \times\left(S^{q} \times S^{q}\right)$, we have one of the following.

(a) $\theta_{*}\left[\{*\} \times S^{q} \times\{*\}\right]=\gamma_{1}$ and $\theta_{*}\left[\{*\} \times\{*\} \times S^{q}\right]=\gamma_{2}$,

(b) $\theta_{*}\left[\{*\} \times S^{q} \times\{*\}\right]=\gamma_{1}+\gamma_{2}$ and $\theta_{*}\left[\{*\} \times\{*\} \times S^{q}\right]=\gamma_{2}$,

(c) $\theta_{*}\left[\{*\} \times S^{q} \times\{*\}\right]=\gamma_{1}$ and $\theta_{*}\left[\{*\} \times\{*\} \times S^{q}\right]=\gamma_{1}+\gamma_{2}$.

Set

$$
X_{1}=\left(S^{1} \times S^{q} \times D^{q+1}\right) \cup_{\theta_{1}} C_{2},
$$

where $\theta_{1}=\theta^{-1}: \partial C_{2} \rightarrow \partial\left(S^{1} \times S^{q} \times D^{q+1}\right)$. Let us consider the Mayer-Vietoris exact sequence

$$
\begin{aligned}
& H_{q+1}\left(X_{1}\right) \rightarrow H_{q}\left(S^{1} \times S^{q} \times S^{q}\right) \\
& \stackrel{\left(j_{1 *},-i_{2 *} \theta_{*}\right)}{\longrightarrow} H_{q}\left(S^{1} \times S^{q} \times D^{q+1}\right) \oplus H_{q}\left(C_{2}\right) \rightarrow H_{q}\left(X_{1}\right),
\end{aligned}
$$

where $j_{1}: S^{1} \times S^{q} \times S^{q} \rightarrow S^{1} \times S^{q} \times D^{q+1}$ is the natural inclusion. In cases (a) and (b), we have that $j_{1 *}\left[\{*\} \times\{*\} \times S^{q}\right]=0, j_{1 *}\left[\{*\} \times S^{q} \times\{*\}\right]$ is a generator of $H_{q}\left(S^{1} \times S^{q} \times D^{q+1}\right)$, and $-i_{2 *} \theta_{*}\left[\{*\} \times\{*\} \times S^{q}\right]$ is a generator of $H_{q}\left(C_{2}\right)$. Hence, the map $\left(j_{1 *},-i_{2 *} \theta_{*}\right)$ is an isomorphism. In cases (a) and (c), we have that $j_{1 *}\left[\{*\} \times S^{q} \times\{*\}\right]$ is a generator of $H_{q}\left(S^{1} \times S^{q} \times D^{q+1}\right),-i_{2 *} \theta_{*}\left[\{*\} \times S^{q} \times\{*\}\right]=0$, 
and $-i_{2 *} \theta_{*}\left[\{*\} \times\{*\} \times S^{q}\right]$ is a generator of $H_{q}\left(C_{2}\right)$. Therefore, again the map $\left(j_{1 *},-i_{2 *} \theta_{*}\right)$ is an isomorphism.

Let us now study the Mayer-Vietoris exact sequence

$$
\begin{aligned}
& H_{q+2}\left(X_{1}\right) \rightarrow H_{q+1}\left(S^{1} \times S^{q} \times S^{q}\right) \\
& \stackrel{\left(j_{1 *},-i_{2 *} \theta_{*}\right)}{\longrightarrow} H_{q+1}\left(S^{1} \times S^{q} \times D^{q+1}\right) \oplus H_{q+1}\left(C_{2}\right) \rightarrow H_{q+1}\left(X_{1}\right) .
\end{aligned}
$$

For this purpose, set

$$
\begin{aligned}
& \widetilde{\gamma}_{1}=a\left[S^{1} \times S^{q} \times\{*\}\right]+b\left[S^{1} \times\{*\} \times S^{q}\right], \\
& \widetilde{\gamma}_{2}=c\left[S^{1} \times S^{q} \times\{*\}\right]+d\left[S^{1} \times\{*\} \times S^{q}\right],
\end{aligned}
$$

which constitute a base of $H_{q+1}\left(S^{1} \times S^{q} \times S^{q}\right)$. Let us show that $i_{1 *} \widetilde{\gamma}_{1}$ is a generator of $H_{q+1}\left(C_{1}\right)$ and $i_{1 *} \widetilde{\gamma}_{2}=0$ in $H_{q+1}\left(C_{1}\right)$.

Since $q$ is odd, we have the intersection numbers

$$
\gamma_{1}^{\prime} \cdot \gamma_{1}^{\prime}=\gamma_{2}^{\prime} \cdot \gamma_{2}^{\prime}=0, \gamma_{1}^{\prime} \cdot \gamma_{2}^{\prime}=-\gamma_{2}^{\prime} \cdot \gamma_{1}^{\prime}= \pm 1
$$

On the other hand, since $i_{1 * \gamma_{2}}=0$ in $H_{q}\left(C_{1}\right)$, there exists a $(q+1)$-chain $\Delta_{2}$ in $C_{1}$ such that $\left[\partial \Delta_{2}\right]=\gamma_{2}$. Then, for the intersection numbers in $C_{1}$, we have

$$
\Delta_{2} \cdot i_{1 *} \widetilde{\gamma}_{1}= \pm 1, \Delta_{2} \cdot i_{1 *} \widetilde{\gamma}_{2}=0
$$

Therefore, we see that $i_{1 *} \widetilde{\gamma}_{1}$ is a generator of $H_{q+1}\left(C_{1}\right)$ and $i_{1 *} \widetilde{\gamma}_{2}=0$.

By a similar argument, we can show that $i_{2} * \widetilde{\gamma}_{2}$ is a generator of $H_{q+1}\left(C_{2}\right)$ and $i_{2 *} \widetilde{\gamma}_{1}=0$.

Then in the exact sequence (2.1), we can show that

$$
\left(j_{1 *},-i_{2 *} \theta_{*}\right): H_{q+1}\left(S^{1} \times S^{q} \times S^{q}\right) \rightarrow H_{q+1}\left(S^{1} \times S^{q} \times D^{q+1}\right) \oplus H_{q+1}\left(C_{2}\right)
$$

is an isomorphism for the cases (a)-(c). Hence, $X_{1}$ is a homotopy $(2 q+2)-$ sphere.

Set $X_{2}=C_{1} \cup_{\theta_{2}} N_{-}$, where $\theta_{2}=\theta: \partial N_{-} \rightarrow \partial C_{1}$ and $N_{-}$is the exterior of the standard embedding of $S^{1} \times S^{q} \times D^{q+1}$ into $S^{2 q+2}$. Let us consider the Mayer-Vietoris exact sequence

$$
H_{k+1}\left(X_{2}\right) \rightarrow H_{k}\left(S^{1} \times S^{q} \times S^{q}\right) \stackrel{\left(i_{1 *} \theta_{*},-j_{2 *}\right)}{\longrightarrow} H_{k}\left(C_{1}\right) \oplus H_{k}\left(N_{-}\right) \rightarrow H_{k}\left(X_{2}\right),
$$

where $j_{2}: S^{1} \times S^{q} \times S^{q} \rightarrow N_{-}$is the natural inclusion. Then, by using the same argument as for $X_{1}$, we can show that $X_{2}$ is also a homotopy $(2 q+2)$-sphere.

Therefore, $f$ has the Fox property. 
(3-2) Let us now consider the case where $q \geq 2$ is even. Let $\eta_{q}$ denote a generator of $H^{q}\left(C_{1}\right) \cong \mathbb{Z}$. Furthermore, let $\left\{\gamma_{1}^{*}, \gamma_{2}^{*}\right\}$ be the base of $H^{q}\left(\partial C_{1}\right) \cong \operatorname{Hom}\left(H_{q}\left(\partial C_{1}\right), \mathbb{Z}\right)$ dual to the base $\left\{\gamma_{1}, \gamma_{2}\right\}$ of $H_{q}\left(\partial C_{1}\right)$. Then, we have

$$
i_{1}^{*} \eta_{q}=u \gamma_{1}^{*}+v \gamma_{2}^{*}
$$

for some integers $u$ and $v$. Since

$$
v=\left\langle i_{1}^{*} \eta_{q}, \gamma_{2}\right\rangle=\left\langle\eta_{q}, i_{1 *} \gamma_{2}\right\rangle=0
$$

holds, we have $i_{1}^{*} \eta_{q}=u \gamma_{1}^{*}$.

Let us consider the cohomology exact sequence for the pair $\left(C_{1}, \partial C_{1}\right)$

$$
H^{q}\left(C_{1}, \partial C_{1}\right) \rightarrow H^{q}\left(C_{1}\right) \stackrel{i_{1}^{*}}{\longrightarrow} H^{q}\left(\partial C_{1}\right) \rightarrow H^{q+1}\left(C_{1}, \partial C_{1}\right) .
$$

Note that we have

$$
H^{q}\left(C_{1}, \partial C_{1}\right) \cong H_{q+2}\left(C_{1}\right)=0 \quad \text { and } \quad H^{q+1}\left(C_{1}, \partial C_{1}\right) \cong H_{q+1}\left(C_{1}\right) \cong \mathbb{Z} .
$$

Therefore, $i_{1}^{*}$ maps $H^{q}\left(C_{1}\right)$ injectively to a nontrivial direct summand of $H^{q}\left(\partial C_{1}\right)$. Therefore, we must have $i_{1}^{*} \eta_{q}= \pm \gamma_{1}^{*}$.

Let $\left\{\left(\gamma_{1}^{\prime}\right)^{*},\left(\gamma_{2}^{\prime}\right)^{*}\right\}$ be the base of $H^{q}\left(S^{q} \times S^{q}\right) \cong \operatorname{Hom}\left(H_{q}\left(S^{q} \times S^{q}\right), \mathbb{Z}\right)$ dual to the base $\left\{\gamma_{1}^{\prime}, \gamma_{2}^{\prime}\right\}$ of $H_{q}\left(S^{q} \times S^{q}\right)$. Then, we have

$$
\begin{aligned}
\left(\gamma_{1}^{\prime}\right)^{*} \smile\left(\gamma_{1}^{\prime}\right)^{*} & =\left(\iota^{*} \gamma_{1}^{*}\right) \smile\left(\iota^{*} \gamma_{1}^{*}\right) \\
& =\iota^{*}\left(\gamma_{1}^{*} \smile \gamma_{1}^{*}\right) \\
& =\iota^{*} i_{1}^{*}\left(\eta_{q} \smile \eta_{q}\right)=0,
\end{aligned}
$$

since $\eta_{q} \smile \eta_{q} \in H^{2 q}\left(C_{1}\right)=0$.

By Poincaré duality, the cup product on $H^{q}\left(S^{q} \times S^{q}\right)$ defines a symmetric bilinear form which is unimodular and of even type. Therefore we have

$$
\left\langle\left(\gamma_{1}^{\prime}\right)^{*} \smile\left(\gamma_{2}^{\prime}\right)^{*},\left[S^{q} \times S^{q}\right]\right\rangle= \pm 1(=\varepsilon),\left\langle\left(\gamma_{2}^{\prime}\right)^{*} \smile\left(\gamma_{2}^{\prime}\right)^{*},\left[S^{q} \times S^{q}\right]\right\rangle=2 m
$$

for some integer $m$. Set

$$
\xi_{2}=\left(\gamma_{2}^{\prime}\right)^{*}-\varepsilon m\left(\gamma_{1}^{\prime}\right)^{*} \in H^{q}\left(S^{q} \times S^{q}\right) .
$$

Then we have

$$
\xi_{2} \smile \xi_{2}=0,\left\langle\left(\gamma_{1}^{\prime}\right)^{*} \smile \xi_{2},\left[S^{q} \times S^{q}\right]\right\rangle=\varepsilon .
$$

Set

$$
\gamma_{1}^{\prime \prime}=\gamma_{1}^{\prime}+\varepsilon m \gamma_{2}^{\prime}, \gamma_{2}^{\prime \prime}=\gamma_{2}^{\prime},
$$


which constitute a base of $H_{q}\left(S^{q} \times S^{q}\right)$. Then we see that the base $\left\{\left(\gamma_{1}^{\prime \prime}\right)^{*},\left(\gamma_{2}^{\prime \prime}\right)^{*}\right\}$ of $H^{q}\left(S^{q} \times S^{q}\right) \cong \operatorname{Hom}\left(H_{q}\left(S^{q} \times S^{q}\right), \mathbb{Z}\right)$ dual to the base $\left\{\gamma_{1}^{\prime \prime}, \gamma_{2}^{\prime \prime}\right\}$ of $H_{q}\left(S^{q} \times S^{q}\right)$ coincides with $\left\{\left(\gamma_{1}^{\prime}\right)^{*}, \xi_{2}\right\}$. Therefore, for the intersection numbers, we have

$$
\gamma_{1}^{\prime \prime} \cdot \gamma_{1}^{\prime \prime}=0, \gamma_{2}^{\prime \prime} \cdot \gamma_{2}^{\prime \prime}=0, \gamma_{1}^{\prime \prime} \cdot \gamma_{2}^{\prime \prime}= \pm 1
$$

Thus, there exists a diffeomorphism $\widehat{\theta}: S^{q} \times S^{q} \rightarrow S^{q} \times S^{q}$ such that

$$
\begin{aligned}
& \theta_{*}\left[\{*\} \times S^{q} \times\{*\}\right]=\iota_{*} \gamma_{1}^{\prime \prime}=\gamma_{1}+\varepsilon m \gamma_{2}, \\
& \theta_{*}\left[\{*\} \times\{*\} \times S^{q}\right]=\iota_{*} \gamma_{2}^{\prime \prime}=\gamma_{2},
\end{aligned}
$$

where $\theta=\operatorname{id}_{S^{1}} \times \widehat{\theta}: S^{1} \times\left(S^{q} \times S^{q}\right) \rightarrow S^{1} \times\left(S^{q} \times S^{q}\right)$.

Since $i_{1 *} \gamma_{2}=0$, there exists a $(q+1)$-chain $\Delta_{2}$ in $C_{1}$ such that $\left[\partial \Delta_{2}\right]=\gamma_{2}$. As

$$
\Delta_{2} \cdot\left(i_{1 *} \theta_{*}\left[S^{1} \times S^{q} \times\{*\}\right]\right)=\gamma_{2} \cdot \theta_{*}\left[S^{1} \times S^{q} \times\{*\}\right]= \pm 1,
$$

we see that $i_{1 *} \theta_{*}\left[S^{1} \times S^{q} \times\{*\}\right]$ generates $H_{q+1}\left(C_{1}\right)$. Furthermore, since

$$
\Delta_{2} \cdot\left(i_{1 *} \theta_{*}\left[S^{1} \times\{*\} \times S^{q}\right]\right)=\gamma_{2} \cdot \theta_{*}\left[S^{1} \times\{*\} \times S^{q}\right]=0,
$$

we see that $i_{1 *} \theta_{*}\left[S^{1} \times\{*\} \times S^{q}\right]=0$ in $H_{q+1}\left(C_{1}\right)$. Then, by the Mayer-Vietoris exact sequence

$$
H_{q+2}\left(S^{2 q+2}\right) \rightarrow H_{q+1}\left(S^{1} \times S^{q} \times S^{q}\right) \rightarrow H_{q+1}\left(C_{1}\right) \oplus H_{q+1}\left(C_{2}\right) \rightarrow H_{q+1}\left(S^{2 q+2}\right),
$$

we see that $i_{2 *} \theta_{*}\left[S^{1} \times\{*\} \times S^{q}\right]$ generates $H_{q+1}\left(C_{2}\right)$.

Then the same argument as in Case (3-1) (b) shows that $f$ has the Fox property.

Case $4 q=1$ and $r=2$

By the same argument as in [11, Section 5], we have only to consider the following two cases.

(4-1) $H_{*}\left(C_{1}\right) \cong H_{*}\left(S^{1} \times S^{1}\right)$,

(4-2) $H_{*}\left(C_{1}\right) \cong H_{*}\left(S^{1} \times S^{2}\right)$.

(4-1) When $H_{*}\left(C_{1}\right) \cong H_{*}\left(S^{1} \times S^{1}\right)$, the natural inclusion

$$
i_{1}^{\prime}: S^{1} \times S^{1} \times\{*\} \rightarrow S^{1} \times S^{1} \times S^{2}=\partial C_{1} \rightarrow C_{1}
$$


induces an isomorphism of homology groups in dimension 1. Then, by considering the commutative diagram

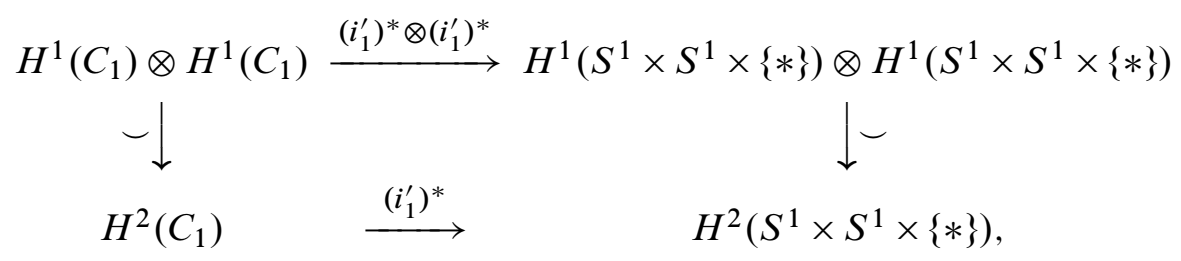

we see that $i_{1}^{\prime}$ induces a homology equivalence.

Using the fact that $2=1+1$ is even, we see that $i_{1 *}\left[\{*\} \times\{*\} \times S^{2}\right]=0$ in $H_{2}\left(C_{1}\right)$. Then by the same argument as before, we can show that $f$ has the Fox property.

(4-2) When $H_{*}\left(C_{1}\right) \cong H_{*}\left(S^{1} \times S^{2}\right)$, by an argument similar to that in the proof of [11, Lemma 5.2], we see that for some diffeomorphism $\widehat{\theta}: S^{1} \times S^{1} \rightarrow S^{1} \times S^{1}$, the composite

$$
\begin{aligned}
& S^{1} \times S^{2} \stackrel{{ }^{\left.\theta\right|_{S}{ }^{1} \times\{*\} \times S^{2}}}{\longrightarrow} S^{1} \times S^{1} \times S^{2}=\partial C_{1} \rightarrow C_{1} \text { or } \\
& S^{1} \times S^{2} \stackrel{{ }_{S^{1} \times\{*\} \times S^{2}}}{\longrightarrow} S^{1} \times S^{1} \times S^{2}=\partial C_{2} \rightarrow C_{2}
\end{aligned}
$$

induces a homology equivalence, where $\theta=\widehat{\theta} \times \operatorname{id}_{S^{2}}:\left(S^{1} \times S^{1}\right) \times S^{2} \rightarrow\left(S^{1} \times S^{1}\right) \times S^{2}$. We may assume that the upper one induces a homology equivalence. By choosing $\widehat{\theta}$ appropriately, we may assume that $\theta_{*}\left[\{*\} \times S^{1} \times\{*\}\right]=0$ in $H_{1}\left(C_{1}\right)$. Then, by the same argument as before, we can show that $f$ has the Fox property.

Case 5 When $1<q, q+1=r$ and $r$ is even.

As in [11, Section 6], we have only to consider the two cases as follows.

(5-1) $\quad H_{*}\left(C_{1}\right) \cong H_{*}\left(S^{1} \times S^{q}\right)$,

(5-2) $\quad H_{*}\left(C_{1}\right) \cong H_{*}\left(S^{1} \times S^{q+1}\right)$ or $H_{*}\left(C_{1}\right) \cong H_{*}\left(S^{q} \times S^{q+1}\right)$.

(5-1) When $H_{*}\left(C_{1}\right) \cong H_{*}\left(S^{1} \times S^{q}\right)$, by the same argument as before, we see that the natural inclusion

$$
i_{1}^{\prime}: S^{1} \times S^{q} \times\{*\} \rightarrow S^{1} \times S^{q} \times S^{q+1}=\partial C_{1} \rightarrow C_{1}
$$


induces isomorphisms of (co)homology groups in dimensions 1 and $q$. By considering the commutative diagram

$$
\begin{aligned}
& H^{1}\left(C_{1}\right) \otimes H^{q}\left(C_{1}\right) \stackrel{\left(i_{1}^{\prime}\right)^{*} \otimes\left(i_{1}^{\prime}\right)^{*}}{\longrightarrow} H^{1}\left(S^{1} \times S^{q}\right) \otimes H^{q}\left(S^{1} \times S^{q}\right) \\
& \smile \downarrow \\
& H^{q+1}\left(C_{1}\right) \quad \stackrel{\left(i_{1}^{\prime}\right)^{*}}{\longrightarrow} \quad H^{q+1}\left(S^{1} \times S^{q}\right),
\end{aligned}
$$

we see that $i_{1}^{\prime}$ induces an isomorphism of (co)homology groups in dimension $q+1$ as well.

Furthermore, since $q+1$ is even, by an argument using the intersection form on $H_{q+1}\left(S^{1} \times S^{q} \times S^{q+1}\right.$ ) (for example, see [11, Lemmas 5.1 and 5.2]), we see that $i_{1 *}\left[\{*\} \times\{*\} \times S^{q+1}\right]=0$. Then, by the Mayer-Vietoris exact sequence

$H_{q+2}\left(S^{2 q+3}\right) \rightarrow H_{q+1}\left(S^{1} \times S^{q} \times S^{q+1}\right) \rightarrow H_{q+1}\left(C_{1}\right) \oplus H_{q+1}\left(C_{2}\right) \rightarrow H_{q+1}\left(S^{2 q+3}\right)$, we see that $i_{2 *}\left[\{*\} \times\{*\} \times S^{q+1}\right]$ is a generator of $H_{q+1}\left(C_{2}\right) \cong \mathbb{Z}$.

Let us consider $X_{1}=\left(S^{1} \times S^{q} \times D^{q+2}\right) \cup_{\theta_{1}} C_{2}$, where $\theta_{1}$ : $\partial C_{2} \rightarrow S^{1} \times S^{q} \times S^{q+1}$ is a natural identification. By the Mayer-Vietoris exact sequence

$H_{k+1}\left(X_{1}\right) \rightarrow H_{k}\left(S^{1} \times S^{q} \times S^{q+1}\right) \rightarrow H_{k}\left(S^{1} \times S^{q} \times D^{q+2}\right) \oplus H_{k}\left(C_{2}\right) \rightarrow H_{k}\left(X_{1}\right)$,

we see that $X_{1}$ is a homotopy $(2 q+3)$-sphere.

Set $X_{2}=C_{1} \cup_{\theta_{2}} N_{-}$, where $N_{-}\left(\simeq S^{q+1} \vee S^{q+2} \vee S^{2 q+1}\right)$ is the exterior of the standard embedding of $S^{1} \times S^{q} \times D^{q+2}$ into $S^{2 q+3}$ and $\theta_{2}: \partial N_{-} \rightarrow \partial C_{1}$ is the natural identification. Then, by the Mayer-Vietoris exact sequence

$$
H_{k+1}\left(X_{2}\right) \rightarrow H_{k}\left(S^{1} \times S^{q} \times S^{q+1}\right) \rightarrow H_{k}\left(C_{1}\right) \oplus H_{k}\left(N_{-}\right) \rightarrow H_{k}\left(X_{2}\right),
$$

we see that $X_{2}$ is also a homotopy $(2 q+3)$-sphere.

Therefore, $f$ has the Fox property.

(5-2) When $H_{*}\left(C_{1}\right) \cong H_{*}\left(S^{1} \times S^{q+1}\right)$ or $H_{*}\left(C_{1}\right) \cong H_{*}\left(S^{q} \times S^{q+1}\right)$, we can show that the natural inclusion

$$
\begin{aligned}
& i_{1}^{\prime}: S^{1} \times\{*\} \times S^{q+1} \rightarrow S^{1} \times S^{q} \times S^{q+1}=\partial C_{1} \rightarrow C_{1} \text { or } \\
& i_{2}^{\prime}:\{*\} \times S^{q} \times S^{q+1} \rightarrow S^{1} \times S^{q} \times S^{q+1}=\partial C_{2} \rightarrow C_{2}
\end{aligned}
$$

induces a homology equivalence, using the fact that $q+1$ is even. Then, by an argument as before, we see easily that $f$ has the Fox property.

This completes the proof of Theorem 1.1. 


\section{A construction}

For $1 \leq p \leq q \leq r$, let $\Sigma^{p, q, r}=\partial N_{+}^{p, q, r}=\partial N^{p}, q, r\left(\cong S^{p} \times S^{q} \times S^{r}\right)$ be the product of three spheres standardly embedded in $S^{p+q+r+1}$ as constructed in Section 1. Then the closure of one of the components of $S^{p+q+r+1} \backslash \Sigma^{p, q, r}$ coincides with $N_{+}^{p, q, r}$, which is diffeomorphic to $S^{p} \times S^{q} \times D^{r+1}$. The other one $N \underline{p}, q, r$, which is diffeomorphic to

$$
\left(\left(\left(D^{p+1} \times S^{r}\right)-\operatorname{Int} D^{p+r+1}\right) \times S^{q}\right) \cup\left(S^{p+r} \times D^{q+1}\right),
$$

is in fact a regular neighborhood of the union of two embedded spheres $S^{p+r}$ and $S^{q+r}$ in $S^{p+q+r+1}$ intersecting each other transversely along $S^{r-1}$. In particular, $N^{p}, q, r$ has the homotopy type of the bouquet $S^{p+r} \vee S^{q+r} \vee S^{r}$.

In [11], we have constructed embeddings $f_{n}: S^{p} \times S^{q} \times S^{r} \rightarrow S^{p+q+r+1}, n \in \mathbb{Z} \backslash\{0\}$, which do not have the Alexander property for $2 \leq p \leq q \leq r$ with $r=p+q$ odd. It is not difficult to observe that exactly the same construction works also for $p=1$ and $r=q+1$ odd. For completeness, let us recall the construction.

Let us write $S^{2 r+1}$ as the union

$$
S^{2 r+1}=\left(D_{-2}^{r+1} \times S^{r}\right) \cup_{\varphi_{-}}\left(S^{r} \times S^{r} \times I\right) \cup_{\varphi_{+}}\left(S^{r} \times D_{2}^{r+1}\right),
$$

where $I=[-1,1], D_{ \pm 2}^{r+1}$ are $(r+1)$-disks, and $\varphi_{-}: \partial\left(D_{-2}^{r+1} \times S^{r}\right) \rightarrow S^{r} \times S^{r} \times\{-1\}$ and $\varphi_{+}: \partial\left(S^{r} \times D_{2}^{r+1}\right) \rightarrow S^{r} \times S^{r} \times\{1\}$ are the standard identification maps. Since $S^{r} \times I$ is diffeomorphic to the closure of the complement of two disjoint $(r+1)$-disks in $S^{r+1}=\left(S^{p} \times D^{q+1}\right) \cup\left(D^{p+1} \times S^{q}\right)$, we can write $S^{r} \times S^{r} \times I$ as the union of $X_{-}=\left(\left(S^{p} \times D^{q+1}\right)-\operatorname{Int} D_{-1}^{r+1}\right) \times S^{r} \quad$ and $\quad X_{+}=\left(\left(D^{p+1} \times S^{q}\right)-\operatorname{Int} D_{1}^{r+1}\right) \times S^{r}$ attached along $S^{p} \times S^{q} \times S^{r}$, which is a boundary component of each, where $D_{ \pm 1}^{r+1}$ are interior disks. Note that the embedding $S^{p} \times S^{q} \times S^{r}=X_{-} \cap X_{+} \subset X_{-} \cup X_{+}=$ $S^{r} \times S^{r} \times I \subset S^{2 r+1}$ defined via (3.1) is standard.

Note that $H_{r}\left(S^{r} \times S^{r}\right)$ is a free abelian group of rank 2 generated by $\left[S^{r} \times\{*\}\right]$ and $\left[\{*\} \times S^{r}\right]$, where we choose orientations of the representative manifolds once and for all. By [4, Proposition 2.5] or [10, Theorem 2.2], for each matrix

$$
\kappa_{n}=\left(\begin{array}{cc}
4 n+1 & 2 n \\
2 & 1
\end{array}\right) \in G L(2, \mathbf{Z})
$$

with $n \in \mathbb{Z} \backslash\{0\}$, the automorphism of $H_{r}\left(S^{r} \times S^{r}\right)=\mathbb{Z} \oplus \mathbb{Z}$ given by the matrix $\kappa_{n}$ with respect to the above base is realized by a diffeomorphism $\psi_{n}: S^{r} \times S^{r} \rightarrow S^{r} \times S^{r}$, since $r$ is odd. By (3.1), we still have

$$
S^{2 r+1} \cong\left(D_{-2}^{r+1} \times S^{r}\right) \cup_{\varphi_{-} \circ \psi_{n}}\left(S^{r} \times S^{r} \times I\right) \cup_{\varphi_{+} \circ \psi_{n}}\left(S^{r} \times D_{2}^{r+1}\right) .
$$


Put $\quad \widetilde{X}_{-}=\left(D_{-2}^{r+1} \times S^{r}\right) \cup_{\varphi_{-} \circ \psi_{n}} X_{-}, \quad \widetilde{X}_{+}=X_{+} \cup_{\varphi_{+} \circ \psi_{n}}\left(S^{r} \times D_{2}^{r+1}\right)$,

and consider the embedding $f_{n}: S^{p} \times S^{q} \times S^{r}=\widetilde{X}_{-} \cap \widetilde{X}_{+} \hookrightarrow \widetilde{X}_{-} \cup \widetilde{X}_{+}=S^{2 r+1}$.

In [11] we have seen $H^{*}\left(\widetilde{X}_{+}\right) \cong H^{*}\left(S^{q} \times S^{r}\right)$ and $H^{*}\left(\widetilde{X}_{-}\right) \cong H^{*}\left(S^{p} \times S^{r}\right)$ as additive groups. Furthermore, the image of the cup product

$$
\smile: H^{q}\left(\widetilde{X}_{+}\right) \otimes H^{r}\left(\widetilde{X}_{+}\right) \rightarrow H^{q+r}\left(\widetilde{X}_{+}\right)
$$

is a subgroup of index $|2 n|$, while that for

$$
\smile: H^{p}\left(\widetilde{X}_{-}\right) \otimes H^{r}\left(\widetilde{X}_{-}\right) \rightarrow H^{p+r}\left(\widetilde{X}_{-}\right)
$$

is a subgroup of index $|4 n+1|$. It is easy to observe that if $f_{n}$ has the Alexander property, then one of the above indices must be equal to 1 or $\infty$. Therefore, we can conclude that the embeddings $f_{n}$ do not have the Alexander property. Note also that if $m \neq n$, then there exists no diffeomorphism of $S^{2 r+1}$ which takes the image of the embedding $f_{n}$ to that of $f_{m}$.

Remark 3.1 For an arbitrary embedding $f: S^{p} \times S^{q} \times S^{r} \rightarrow S^{p+q+r+1}, 1 \leq p \leq q \leq$ $r$, let us denote by $C_{1}$ and $C_{2}$ the closures of the two components of $S^{p+q+r+1} \backslash \operatorname{Im} f$. Then, as has been observed in [11], $C_{1}$ or $C_{2}$ has the homology of $S^{p} \times S^{q}, S^{p} \times S^{r}$ or $S^{q} \times S^{r}$.

When $r=p+q$ is odd, for the embeddings $f_{n}$ constructed above, the manifolds $C_{1}$ and $C_{2}$ have the homologies of $S^{p} \times S^{r}$ and $S^{q} \times S^{r}$. Recall that by [11, Proposition 7.1] if $H_{*}\left(C_{1}\right) \cong H_{*}\left(S^{p} \times S^{q}\right), p, q \geq 2$, then $C_{1}$ is diffeomorphic to $S^{p} \times S^{q} \times D^{r+1}$ : consequently, such an $f$ has the Alexander property.

In Section 5, we will prove the following.

Theorem 3.2 Suppose $1 \leq p \leq q \leq r$, and $r=p+q$ is odd. Then the smooth embeddings $f_{n}: S^{p} \times S^{q} \times S^{r} \rightarrow S^{p+q+r+1}, n \neq 0,-1$, do not have the Fox property in the smooth category.

Remark 3.3 In [12], embeddings of $S^{1} \times S^{q} \times S^{r}$ into $S^{q+r+2}$ whose images do not bound the product of two spheres and a disk have been constructed. However, they do have the Alexander property, since the closure of one of the two components of the complement is diffeomorphic to the closure of one of the two components of the complement of a standard embedding. Thus, in a sense, the embeddings constructed for $p=1$ in [12] are weakly knotted. We do not know if there exist embeddings $S^{1} \times S^{q} \times S^{r} \rightarrow S^{q+r+2}$ that do not have the Alexander property for general $q$ and $r$. (As has been mentioned above, such embeddings do exist if $r=q+1$ is odd.) 


\section{Diffeomorphisms of $S^{p} \times S^{q} \times S^{r}$ for $r=p+q$}

For the proof of Theorem 3.2, we need a lemma concerning diffeomorphisms of $S^{p} \times S^{q} \times S^{r}$ with $r=p+q$ and $p \neq q$.

Set $M=S^{p} \times S^{q} \times S^{r}$ with $r=p+q$. Let us study those automorphisms of $H_{r}(M) \cong \mathbb{Z} \oplus \mathbb{Z}$ which are induced by diffeomorphisms of $M$. Set

$$
\alpha_{1}=\left[S^{p} \times S^{q} \times\{*\}\right], \quad \alpha_{2}=\left[\{*\} \times\{*\} \times S^{r}\right],
$$

which constitute a base of $H_{r}(M)$. To each diffeomorphism $\varphi: M \rightarrow M$ we associate the $2 \times 2$ integer matrix $\left[\varphi_{*}\right]=\left(a_{i j}\right) \in G L(2, \mathbb{Z})$ by

$$
\varphi_{*} \alpha_{j}=\sum_{i=1}^{2} a_{i j} \alpha_{i}, \quad j=1,2 .
$$

Lemma 4.1 The matrix $\left[\varphi_{*}\right]$ is of the form

$$
\left(\begin{array}{cc} 
\pm 1 & 0 \\
* & \pm 1
\end{array}\right)
$$

Proof Let $\eta_{p} \in H^{p}(M)$ and $\eta_{q} \in H^{q}(M)$ be the cohomology classes Poincaré dual to the homology classes $\left[\{*\} \times S^{q} \times S^{r}\right] \in H_{q+r}(M)$ and $\left[S^{p} \times\{*\} \times S^{r}\right] \in H_{p+r}(M)$, respectively. Note that $H^{p}(M)$ and $H^{q}(M)$ are infinite cyclic groups generated by $\eta_{p}$ and $\eta_{q}$, respectively. Since $\varphi^{*}: H^{*}(M) \rightarrow H^{*}(M)$ is an automorphism, we have $\varphi^{*} \eta_{p}= \pm \eta_{p}$ and $\varphi^{*} \eta_{q}= \pm \eta_{q}$. In particular, we have

$$
\varphi^{*}\left(\eta_{p} \smile \eta_{q}\right)= \pm \eta_{p} \smile \eta_{q} \in H^{p+q}(M)=H^{r}(M) .
$$

Let $\xi_{1}$ and $\xi_{2} \in H^{r}(M)$ be the cohomology classes Poincare dual to the homology classes $\left[\{*\} \times\{*\} \times S^{r}\right]$ and $\left[S^{p} \times S^{q} \times\{*\}\right] \in H_{r}(M)$, respectively. Note that we may assume $\eta_{p} \smile \eta_{q}=\xi_{1}$, since $\left(\{*\} \times S^{q} \times S^{r}\right) \cap\left(S^{p} \times\{*\} \times S^{r}\right)=\{*\} \times\{*\} \times S^{r}$. Note also that $\left\{\xi_{1}, \xi_{2}\right\}$ is the base of $H^{r}(M) \cong \operatorname{Hom}\left(H_{r}(M), \mathbb{Z}\right)$ dual to $\left\{\alpha_{1}, \alpha_{2}\right\}$. Then the matrix representative of the automorphism $\varphi^{*}: H^{r}(M) \rightarrow H^{r}(M)$ with respect to the base $\left\{\xi_{1}, \xi_{2}\right\}$ is of the form

$$
\left(\begin{array}{cc} 
\pm 1 & * \\
0 & \pm 1
\end{array}\right)
$$

Therefore, $\varphi_{*}: H_{r}(M) \rightarrow H_{r}(M)$ has a matrix representative, with respect to the dual base $\left\{\alpha_{1}, \alpha_{2}\right\}$, of the form

$$
\left(\begin{array}{cc} 
\pm 1 & 0 \\
* & \pm 1
\end{array}\right)
$$

This completes the proof. 


\section{Proof of Theorem 3.2}

Let $C_{1}$ and $C_{2}$ be the closures of the two components of $S^{p+q+r+1} \backslash f_{n}\left(S^{p} \times S^{q} \times S^{r}\right)$. We may assume

$$
H_{*}\left(C_{1}\right) \cong H_{*}\left(S^{p} \times S^{r}\right), \quad H_{*}\left(C_{2}\right) \cong H_{*}\left(S^{q} \times S^{r}\right) .
$$

Note that in the notation of Section 3 or [11, Section 9], $C_{1}$ and $C_{2}$ correspond to $\widetilde{X}_{-}$ and $\widetilde{X}_{+}$, respectively.

Our strategy is to show that the closed manifold $C_{i} \cup N_{ \pm}^{p^{\prime}, q^{\prime}, r^{\prime}}$ obtained by attaching $C_{i}$ and $N_{ \pm}^{p^{\prime}, q^{\prime}, r^{\prime}}$ along their boundaries, $i=1,2$, can never be diffeomorphic to the sphere $S^{\frac{ \pm}{p+q+r+1}}$ for every permutation $\left(p^{\prime}, q^{\prime}, r^{\prime}\right)$ of $(p, q, r)$.

Let us first show that $C_{1}$ cannot be diffeomorphic to the exterior of $N_{+}^{p^{\prime}, q^{\prime}, r^{\prime}}$ embedded in $S^{p+q+r+1}$. Suppose this was the case. Then, by an argument using the Alexander duality, we see that the manifold

$$
W=C_{1} \cup_{\varphi}\left(D^{p+1} \times S^{q} \times S^{r}\right),
$$

obtained by attaching $D^{p+1} \times S^{q} \times S^{r}$ to $C_{1}$ by using a diffeomorphism $\varphi: \partial\left(D^{p+1} \times\right.$ $\left.S^{q} \times S^{r}\right) \rightarrow \partial C_{1}$, should be diffeomorphic to $S^{p+q+r+1}$.

Let us consider the Mayer-Vietoris exact sequence associated with the decomposition

$$
H_{r}\left(S^{p} \times S^{q} \times S^{r}\right) \stackrel{\Phi=\left(\theta_{*},-j_{*}\right)}{\longrightarrow} H_{r}\left(C_{1}\right) \oplus H_{r}\left(D^{p+1} \times S^{q} \times S^{r}\right) \longrightarrow H_{r}(W),
$$

where $j: S^{p} \times S^{q} \times S^{r}=\partial\left(D^{p+1} \times S^{q} \times S^{r}\right) \rightarrow D^{p+1} \times S^{q} \times S^{r}$ is the inclusion map and $\theta: S^{p} \times S^{q} \times S^{r} \rightarrow C_{1}$ is given by the composition

$$
\theta: S^{p} \times S^{q} \times S^{r}=\partial\left(D^{p+1} \times S^{q} \times S^{r}\right) \stackrel{\varphi}{\longrightarrow} S^{p} \times S^{q} \times S^{r}=\partial C_{1} \stackrel{i_{1}}{\longrightarrow} C_{1}
$$

with $i_{1}: \partial C_{1} \rightarrow C_{1}$ being the inclusion map.

By Lemma 4.1, we have $\varphi_{*} \alpha_{1}= \pm \alpha_{1}+b \alpha_{2}$ for some $b \in \mathbb{Z}$. Let $\beta \in H_{r}\left(C_{1}\right) \cong \mathbb{Z}$ be a generator. Furthermore, set $\beta^{\prime}=\left[\{*\} \times\{*\} \times S^{r}\right] \in H_{r}\left(D^{p+1} \times S^{q} \times S^{r}\right) \cong \mathbb{Z}$, which is a generator. Then, by the construction of $f_{n}$, we have $i_{1 *} \alpha_{1}= \pm 2 \beta$ and $i_{1 *} \alpha_{2}= \pm(4 n+1) \beta$ (for details, see [11]). Therefore, we have

$$
\theta_{*} \alpha_{1}=i_{1 *} \varphi_{*} \alpha_{1}=i_{1 *}\left( \pm \alpha_{1}+b \alpha_{2}\right)= \pm 2 \beta \pm b(4 n+1) \beta=( \pm 2 \pm b(4 n+1)) \beta .
$$

On the other hand, by Lemma 4.1, we have $\varphi_{*} \alpha_{2}= \pm \alpha_{2}$. Hence, we have

$$
\theta_{*} \alpha_{2}=i_{1 *} \varphi_{*} \alpha_{2}=i_{1 *}\left( \pm \alpha_{2}\right)= \pm(4 n+1) \beta .
$$


Furthermore, we have $j_{*} \alpha_{1}=0$, since $\alpha_{1}=\left[S^{p} \times S^{q} \times\{*\}\right]$ and $S^{p} \times S^{q} \times\{*\}=$ $\partial\left(D^{p+1} \times S^{q} \times\{*\}\right)$. We also have $j_{*} \alpha_{2}= \pm \beta^{\prime}$.

Therefore, the matrix representative of $\Phi=\left(\theta_{*},-j_{*}\right)$ in (5.2) with respect to the bases $\left\{\alpha_{1}, \alpha_{2}\right\}$ and $\left\{\beta, \beta^{\prime}\right\}$ is of the form

$$
\left(\begin{array}{cc} 
\pm 2 \pm b(4 n+1) & \pm(4 n+1) \\
0 & \pm 1
\end{array}\right)
$$

According to the exact sequence (5.2), in order to have $H_{r}(W)=0$, we must have

$$
\pm 2 \pm b(4 n+1)= \pm 1
$$

for some integer $b$, which is possible only when $n=0,-1$. As we have assumed $n \neq 0,-1$, this is impossible. Consequently, the manifold $W$ cannot be diffeomorphic to $S^{p+q+r+1}$.

Let us now show that $C_{1}$ cannot be diffeomorphic to the exterior of $N_{-}=N^{p^{\prime}}, q^{\prime}, r^{\prime}$ embedded in $S^{p+q+r+1}$. For this, let us consider the manifold

$$
W^{\prime}=C_{1} \cup_{\varphi^{\prime}} N_{-},
$$

obtained by attaching $N_{-}$to $C_{1}$ by using a diffeomorphism $\varphi^{\prime}: \partial N_{-} \rightarrow \partial C_{1}$. Note that by an argument using the Alexander duality, we have only to consider the case where $N_{-}$is the exterior of $S^{p} \times S^{r}$ standardly embedded in $S^{p+q+r+1}$ :

$N_{-}=\left(\left(\left(D^{p+1} \times S^{q}\right)-\operatorname{Int} D^{p+q+1}\right) \times S^{r}\right) \cup\left(S^{p+q} \times D^{r+1}\right) \simeq S^{p+q} \vee S^{q+r} \vee S^{q}$

Let us consider the Mayer-Vietoris exact sequence

$$
H_{r}\left(S^{p} \times S^{q} \times S^{r}\right) \stackrel{\Phi^{\prime}=\left(\theta_{*}^{\prime},-j_{*}^{\prime}\right)}{\longrightarrow} H_{r}\left(C_{1}\right) \oplus H_{r}\left(N_{-}\right) \longrightarrow H_{r}\left(W^{\prime}\right),
$$

where $j^{\prime}: S^{p} \times S^{q} \times S^{r}=\partial N_{-} \rightarrow N_{-}$is the inclusion map and $\theta^{\prime}: S^{p} \times S^{q} \times S^{r} \rightarrow C_{1}$ is given by the composition

$$
\theta^{\prime}: S^{p} \times S^{q} \times S^{r}=\partial N_{-} \stackrel{\varphi^{\prime}}{\longrightarrow} S^{p} \times S^{q} \times S^{r}=\partial C_{1} \stackrel{i_{1}}{\longrightarrow} C_{1} .
$$

By an argument similar to the above, we can show that a matrix representative of $\Phi^{\prime}$ is of the form

$$
\left(\begin{array}{cc} 
\pm 2 \pm b(4 n+1) & \pm(4 n+1) \\
\pm 1 & 0
\end{array}\right)
$$

for some integer $b$. Therefore, we have $H_{r}\left(W^{\prime}\right)=0$ only if $\pm(4 n+1)= \pm 1$, which is possible only if $n=0$. Since we have assumed $n \neq 0$, this shows that $W^{\prime}$ cannot be diffeomorphic to $S^{p+q+r+1}$.

Consequently, the embedding $f_{n}, n \neq 0,-1$, does not have the Fox property. This completes the proof of Theorem 3.2. 
Remark 5.1 In [11, Section 9], we have used the matrix $\kappa_{n}$ as in (3.2) for constructing the embeddings $f_{n}$. Analyzing the proof, we see that we could as well use the matrix

$$
\left(\begin{array}{cc}
4 n+1 & 8 n \\
2 n & 4 n-1
\end{array}\right)
$$

for the construction. Let the resulting embedding be denoted by $g_{n}: S^{p} \times S^{q} \times S^{r} \rightarrow$ $S^{p+q+r+1}$. Then we can show that for $g_{n}, n \neq 0,-1$, the closures $C_{1}$ and $C_{2}$ of the two components of $S^{p+q+r+1} \backslash \operatorname{Im} g_{n}$ both violate the Fox property. In other words, we can show that none of $C_{1} \cup\left(D^{p+1} \times S^{q} \times S^{r}\right), C_{1} \cup N^{p}, r, q,\left(S^{p} \times D^{q+1} \times S^{r}\right) \cup C_{2}$ or $N^{q}, r, p \cup C_{2}$ is diffeomorphic to $S^{p+q+r+1}$.

In this paper, we have mainly worked in the smooth category. By using similar techniques, we can show that Theorems 1.1 and 3.2 are valid in the PL locally flat category as well. The proofs are almost the same: it suffices to replace diffeomorphisms by PL homeomorphisms. We also need the solution to the high dimensional PL Poincaré conjecture proved by Smale (see [14], for example).

\section{Summary of results and open problems}

Summarizing the results obtained so far, for a smooth embedding $f: S^{p} \times S^{q} \times S^{r} \rightarrow$ $S^{p+q+r+1}$ with $1 \leq p \leq q \leq r$, we have the following.

(i) When $2 \leq p$, and either $p+q \neq r$ or $p+q=r$ is even, $f$ has the Alexander property [11]. In fact, its image always bounds the product of two spheres and a disk.

(ii) When $p=1,3 \leq q+r$, and either $1+q \neq r$ or $1+q=r$ is even, $f$ has the Fox property (Theorem 1.1 of the present paper).

(iii) When $(p, q, r)=(1,1,1), f$ has the Fox property in the TOP locally flat category [12].

(iv) When $p=1$, there exist infinitely many embeddings such that their images do not bound the product of two spheres and a disk, but that they all have the Alexander property [12].

(v) When $p+q=r$ is odd, there exist infinitely many smooth embeddings which do not have the Fox property ([11] and Theorem 3.2 of the present paper).

We end this paper by posing some problems. 
Problem 6.1 (1) Characterize the exteriors of smooth embeddings $f: S^{p} \times S^{q} \times S^{r} \rightarrow$ $S^{p+q+r+1}$, especially when they do not have the Fox property.

(2) Let $S^{p+q+r+1}=N_{+} \cup N_{-}$be the decomposition induced by a standard embedding of $S^{p} \times S^{q} \times S^{r}$ into $S^{p+q+r+1}$. Given arbitrary two smooth embeddings $\varphi_{ \pm}: N_{ \pm} \rightarrow$ $S^{p+q+r+1}$, does there exist a smooth embedding $f: S^{p} \times S^{q} \times S^{r} \rightarrow S^{p+q+r+1}$ such that the closures of the two components of $S^{p+q+r+1} \backslash \operatorname{Im} f$ are diffeomorphic to the exteriors of $\varphi_{ \pm}$?

Acknowledgements This work is part of the Projeto Temático - Topologia Algébrica, Diferencial e Geométrica - FAPESP, 2008/57607-6.

The second author has been supported in part by Grant-in-Aid for Scientific Research (No. 19340018 and No. 23244008), Japan Society for the Promotion of Science.

The authors would like to thank the referee for many important comments that drastically improved the presentation of the paper. In particular, the notion of Alexander property is due to him/her. They would also like to thank Prof. Oziride Manzoli Neto for stimulating discussions and his constant encouragement.

\section{References}

[1] J W Alexander, On the subdivision of a 3-space by a polyhedron, Proc. Nat. Acad. Sci. USA 10 (1924) 6-8

[2] M Brown, A proof of the generalized Schoenflies theorem, Bull. Amer. Math. Soc. 66 (1960) 74-76 MR0117695

[3] R H Fox, On the imbedding of polyhedra in 3-space, Ann. of Math. (2) 49 (1948) 462-470 MR0026326

[4] $\mathbf{R} Z$ Goldstein, Piecewise linear unknotting of $S^{p} \times S^{q}$ in $S^{p+q+1}$, Michigan Math. J. 14 (1967) 405-415 MR0220299

[5] A Katanaga, O Saeki, Embeddings of quaternion space in $S^{4}$, J. Austral. Math. Soc. Ser. A 65 (1998) 313-325 MR1660419

[6] M A Kervaire, J W Milnor, Groups of homotopy spheres. I, Ann. of Math. (2) 77 (1963) 504-537 MR0148075

[7] S Kinoshita, On Fox's property of a surface in a 3-manifold, Duke Math. J. 33 (1966) 791-794 MR0203720

[8] A Kosiński, On Alexander's theorem and knotted spheres, from: "Topology of 3manifolds and related topics (Proc. The Univ. of Georgia Institute, 1961)", Prentice-Hall, Englewood Cliffs, N.J. (1962) 55-57 MR0158369 
[9] L A Lucas, O M Neto, O Saeki, A generalization of Alexander's torus theorem to higher dimensions and an unknotting theorem for $S^{p} \times S^{q}$ embedded in $S^{p+q+2}$, Kobe J. Math. 13 (1996) 145-165 MR1442202

[10] L A Lucas, O Saeki, Diffeomorphisms of a product of spheres and embedded spheres, Topology Appl. 123 (2002) 471-478 MR1924046

[11] L A Lucas, O Saeki, Embeddings of $S^{p} \times S^{q} \times S^{r}$ in $S^{p+q+r+1}$, Pacific J. Math. 207 (2002) 447-462 MR1972255

[12] L A Lucas, O Saeki, Codimension one embeddings of product of three spheres, Topology Appl. 146/147 (2005) 409-419 MR2107160

[13] J H Rubinstein, Dehn's lemma and handle decompositions of some 4-manifolds, Pacific J. Math. 86 (1980) 565-569 MR590570

[14] S Smale, Generalized Poincaré's conjecture in dimensions greater than four, Ann. of Math. (2) 74 (1961) 391-406 MR0137124

[15] S Suzuki, On a complexity of a surface in 3-sphere, Osaka J. Math. 11 (1974) 113-127 MR0346791

[16] C T C Wall, Killing the middle homotopy groups of odd dimensional manifolds, Trans. Amer. Math. Soc. 103 (1962) 421-433 MR0139185

[17] C T C Wall, Unknotting tori in codimension one and spheres in codimension two, Proc. Cambridge Philos. Soc. 61 (1965) 659-664 MR0184249

Academia da Força Aérea, Alameda dos Pinheiros, 4315, 13632-490 Pirassununga, SP, Brazil Institute of Mathematics for Industry, Kyushu University, Motooka 744, Nishi-ku, Fukuoka 819-0395, Japan

laercio.lap@gmail.com, saeki@imi.kyushu-u.ac.jp

http://imi.kyushu-u.ac.jp/ saeki/

Received: 26 February $2011 \quad$ Revised: 3 October 2011 